ISSN: 0210-1696

DOI: http://dx.doi.org/10.14201/scero20174826179

\title{
JUGAR Y CONSTRUIR: EXPERIENCIAS DE PARTICIPACIÓN DE NIÑOS CON DISCAPACIDAD INTELECTUAL DE SEGUNDO CICLO BÁSICO EN DOS ESCUELAS MUNICIPALES EN CHILE
}

\section{Playing and building: experiences of participation of children with intellectual disability of second basic cycle in two municipal schools in Chile}

\author{
Rodolfo Israel Soto GonZÁLEZ \\ Universidad de Chile. José Joaquín Pérez \#1384 \\ San Bernardo. Santiago de Chile. Chile \\ rodolfosotogo@gmail.com \\ Mauricio Andrés López CRUZ \\ Universidad de Chile. Departamento de Psicología
}

Recepción: 26 de enero de 2017

Aceptación definitiva: 12 de mayo de 2017

RESUMEN: La participación de los niños con discapacidad intelectual [DI] es promovida desde el enfoque de derechos y la educación inclusiva. No obstante, en Chile estos niños tienden a ser segregados por los programas educativos existentes. El objetivo de este estudio corresponde a comprender las experiencias de participación de niños con DI en el contexto escolar regular. Para responder a tal objetivo, se realizaron entrevistas individuales a 15 niños con DI que cursaban segundo ciclo básico en dos escuelas de la Región Metropolitana de Chile. La producción de información se apoyó en técnicas narrativo-visuales tales como fotografía participativa y entrevistas episódicas en combinación con foto-elicitación. El análisis se realizó en base a la Teoría Fundamentada. Los resultados muestran que las experiencias de participación se dan principalmente en 
dos tipos de actividad: 1) actividades didácticas que suponen la construcción de material físico y/o simbólico y 2) juegos de carácter grupal. Estos resultados permiten identificar, desde la perspectiva de los niños con DI, recursos y apoyos universales presentes en el medio comunitario escolar para promover su participación, motivación, aprendizaje y calidad de vida.

Palabras Clave: participación; discapacidad intelectual; inclusión educativa; juego.

Aвstract: Participation of children with intellectual disabilities [ID] is promoted from a rights-based approach and inclusive education. However, in Chile these children tend to be segregated by existing educational programs. The objective of this study is to understand the experiences of participation of children with ID in the regular school context. In order to respond to this objective, individual interviews were conducted with 15 children with mild ID who attended the second basic cycle in two schools in the Metropolitan Region of Chile. Production of information was based on narrative-visual techniques such as participatory photography and episodic interviews in combination with photo-elicitation. The analysis was based on the Grounded Theory. The results show that the experiences of participation take place mainly in two types of activities: 1) didactic activities that involve the construction of physical and/or symbolic material and 2) games of a group character. These results allow the identification, from the perspective of children with ID, of universal resources and supports existing in the school community environment to promote their participation, motivation, learning and quality of life.

KEYWORDS: participation; intellectual disability; educational inclusion; play.

\section{Introducción}

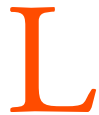

A PARTICIPACIÓN FIGURA EN LOS PRINCIPALES TRATADOS DE DERECHOS HUMANOS en múltiples formas (ONU, 2006a), asumiendo un importante papel para promover la dignidad humana. Así, por ejemplo, la Convención sobre las Personas con Discapacidad estipula que la discapacidad resulta de barreras que obstaculizan la plena participación en la sociedad (ONU, 2006b). Por su parte, la Convención sobre los Derechos del Niño reconoce el derecho a ser escuchado (ONU, 1986), siendo su ejercicio permanente comprendido como participación (Comité de los Derechos del Niño, 2009). Aun con esta base normativa, los niños con discapacidad siguen siendo un grupo particularmente limitado en materia de participación (Unicef, 2013). A muchos se les abandona en instituciones donde se merma su participación social y a otros, inclusive, se les niega la educación (Lansdown, 2005).

La participación juega un papel importante en el ámbito educativo, ya que el acceso a la escuela por sí solo es insuficiente para incrementar la inclusión de todos (Booth y Ainscow, 2011). En efecto, fomentar la participación conlleva la inclusión de los niños en los procesos sociales de su comunidad educativa, generando mayores oportunidades de aprendizaje (Unicef, 2013). De esta manera, es posible conseguir 
mayores niveles de logro educativo mediante la promoción de la participación (BlackHawkins, Florian y Rouse, 2008). La participación debe estar bajo el contexto de la propia comunidad educativa y en igualdad de condiciones con los demás. En otras palabras, las prácticas educativas deben basarse en los principios del diseño universal, confeccionando productos, entornos, programas y servicios que puedan utilizar todas las personas, en la mayor medida posible, sin necesidad de diseño especializado (ONU, 2006a).

Múltiples perspectivas actuales de la participación son coherentes con el criterio de la universalidad, puesto que destacan el papel de las actividades comunitarias y sus consecuencias favorables en las personas que participan en ellas. En esta línea, la European Agency for Development in Special Needs Education entiende la participación como la implicación en actividades típicas con alta prioridad social, prioridad derivada desde la importancia para el individuo, su pertinencia para objetivos sociales y especialmente el nivel de extensión de su práctica en el grupo etario (2011). En estas actividades, los niños desarrollan sus facultades mentales mediante una participación en forma de protagonismo activo que conlleva la asunción de responsabilidades y la realización de contribuciones (Lansdown, 2005). Es más, Rogoff (1997) asevera que las personas se transforman mediante los procesos interpersonales que las implican en una actividad organizada y valorada socioculturalmente, pudiendo manejar situaciones ulteriores. Asimismo, Wenger (2002) arguye que la participación en prácticas comunes, al interior de la misma comunidad, permite la construcción de identidad y sentido de pertenencia entre sus miembros y que el involucramiento en actividades significativamente distintas genera una segregación identitaria. Por último, Verdugo, Navas, Gómez y Schalock (2012) dan cuenta que la calidad de vida está sujeta, entre otros aspectos, a la oportunidad de perseguir el enriquecimiento de su vida en los principales escenarios de actividad.

Estos planteamientos pueden sintetizarse en una concepción de la participación que la comprende como un proceso social donde se reconocen las perspectivas y acciones de las personas al considerarlas como contribuciones en el contexto de una actividad socialmente valorada, propiciando mayores niveles de calidad de vida, identidad positiva y desarrollo.

Asimismo, la participación es un constructo de alta preeminencia en la comprensión de la discapacidad intelectual [DI]. La Asociación Americana de Discapacidades Intelectuales y del Desarrollo [AADID], al abandonar el concepto de retraso mental, que considera la discapacidad como una condición interna de la persona, posiciona la participación como una dimensión clave en la definición constitutiva de la DI. Este cambio se produce al otorgar un mayor énfasis a una perspectiva ecológica, la que se centra en la participación de la persona con su entorno (AADID, 2011). En la trayectoria histórica de la AADID, ha crecido gradualmente la importancia del concepto de participación en el marco de su modelo explicativo (Verdugo, Schalock, Thompson y Guillén, 2013). Es así como distintos modelos de planificación e intervención para personas con discapacidades destacan el rol de la participación, tales como el Modelo de Calidad de Vida (Verdugo, Navas, Gómez y Schalock, 2012) o el Modelo de Apoyos para personas con discapacidades intelectuales y del desarrollo (AADID, 2011). 
En Chile, con la promulgación e implementación del Decreto 170, se ha avanzado en el acceso a la educación regular de los niños con DI. No obstante, esta apertura se focaliza a niños con DI que presentan un cociente intelectual de 50-69, acaparando las escuelas especiales al resto de los estudiantes con esta discapacidad. Los niños con DI representan el segundo diagnóstico más frecuente bajo la cobertura de este decreto y, en conjunto con los niños diagnosticados con funcionamiento intelectual limítrofe -diagnóstico con similares características según la AADID (2011)-, se posicionan en el primer lugar. Además, a medida que disminuye el nivel socioeconómico incrementa la proporción de niños con DI, siendo las escuelas municipales las que mayoritariamente acogen estos estudiantes (Fundación Chile, 2013). Las escuelas municipales concentran a los estudiantes de nivel socioeconómico más bajo y disponen de un índice de vulnerabilidad escolar mayor a los establecimientos particulares subvencionados (JUNAEB, 2015). Cabe agregar que, en Chile, la discapacidad de índole intelectual es el diagnóstico más recurrente en el periodo escolar, si es comparada con otros tipos de discapacidad (Fondo Nacional de Discapacidad, 2005). En conjunto, estos datos revelan la trascendencia de este grupo escolar.

El Decreto 170 permite entregar recursos financieros adicionales por cada niño con DI u otra necesidad educativa especial diagnosticada, para apoyar el proceso educativo con la contratación de especialistas y adquisición de materiales pedagógicos (Ministerio de Educación [MINEDUC], 2009a). De esta manera, se constituyen los Programas de Integración Escolar [PIE], que son caracterizados por el MINEDUC (2009b) como una estrategia “inclusiva”. No obstante, López, Julio, Pérez, Morales y Rojas (2014) describen la situación de la integración escolar en Chile como un sistema híbrido en el que, a nivel de política educativa, se interpela a la inclusión social desde la lógica de los derechos humanos, pero prescribe un modelo de integración con un fuerte énfasis psicomédico, que descansa en una visión patológica de la diferencia. Este planteamiento se centra en el diagnóstico y el tratamiento individualizado ofrecido por el docente especializado. Por lo tanto, el proceso recae en el etiquetado y la segregación, creando una barrera cultural que sitúa las posibilidades de cambio y aprendizaje fuera del ámbito educativo regular.

Esta respuesta educativa hace que los niños usuarios del PIE permanezcan en mayor grado con los especialistas y en menor medida con sus compañeros, profesores de aula regular o en experiencias comunes (Fundación Chile, 2013). Este trato diferenciado por parte de la comunidad hacia los niños que participan en el PIE incrementa su estigmatización y eventualmente su victimización (Villalobos et al., 2014). Específicamente para los estudiantes con DI, la influencia que posee la aplicación de respuestas educativas distintas en función de su diagnóstico que incluye su adscripción al PIE, trasciende su estadía en la escuela al impactar en sus proyectos de vida. En efecto, sus proyectos de vida se caracterizan por la internalización de bajas expectativas en sus propias capacidades producto de la experiencia de discriminación y segregación vivida (López, 2015). Se demuestran, con estas aportaciones, las perversas secuelas originadas al menguar las experiencias de participación de los niños con DI en sus entornos "naturales".

Estos antecedentes revelan también la escasa importancia atribuida a la participación como medio para estimular la inclusión y el desarrollo de los niños con DI que 
son parte de los PIE en escuelas regulares. No obstante, los estudiantes catalogados con necesidades educativas especiales manifiestan necesidades de apoyo donde destacan la participación, el aprendizaje y las relaciones interpersonales con sus compañeros (Woolfson, Harker, Lowe, Sheilds y Mackintosh, 2007). En este sentido, es necesario escuchar la voz de los niños, dado que la creación de escuelas inclusivas es posible fundamentalmente a través de la recolección de sus opiniones (Fielding, 2011).

En esta dirección se origina el presente estudio, al establecer como su objetivo general comprender las experiencias de participación de niños con DI en el contexto escolar regular. Como objetivos específicos se plantean dos: a) identificar actividades socialmente valoradas en el contexto escolar regular desde la perspectiva de niños con discapacidad intelectual y b) describir ideas de los niños con discapacidad intelectual sobre la participación en las actividades socialmente valoradas del escenario escolar. Responder estos objetivos aportaría a entender la participación desde la perspectiva de los niños con DI, lo que ayudaría a impulsar apoyos acertados y suprimir barreras, permitiendo el mejoramiento de su desarrollo, la garantía de sus derechos y la construcción de escuelas y programas educativos inclusivos.

\section{Método}

Se utilizó un diseño de investigación cualitativo, dado que presenta mayores ventajas para abordar las experiencias de los niños con DI en su contexto escolar como objeto de estudio. Precisamente, la metodología cualitativa tiene su foco en las perspectivas, significados y visiones de los participantes en sus contextos sociales (Flick, 2007), permitiendo mayor profundidad en la comprensión de las "experiencias" (Silverman, 2000). Las experiencias fueron producidas con técnicas narrativo-visuales, ya que dichas técnicas permiten favorecer la voz de los niños en torno a su participación en el medio escolar (López, Valdivia y Fernández, 2016).

\subsection{Participantes}

Los participantes de este estudio pertenecían a dos escuelas de educación básica con modalidad regular ubicadas en la Región Metropolitana de Chile. Estas escuelas tienen asignado un índice de vulnerabilidad escolar (IVE-SINAE) mayor al 80\%. Este índice es construido con variables asociadas a la condición socioeconómica, sirviendo como indicador de pobreza al presentarse puntuaciones altas (Junaeb, 2005). Se descartaron las escuelas especiales, ya que las experiencias de participación debían darse en actividades escolares socialmente valoradas, las que incluyen la diversidad inherente a las comunidades humanas. Igualmente, se optó por centros educativos que dispusieran de un PIE vigente, pues cuentan con los documentos en torno al diagnóstico de DI. Como resultado de realizar el estudio en escuelas regulares con PIE, los participantes contaban con un diagnóstico de DI dentro del rango 50-69 de cociente intelectual. 
Los niños participantes pertenecen al segundo ciclo de educación básica, teniendo una edad de entre 10 y 14 años. Fueron en total 15 niños (7 hombres y 8 mujeres), cifra que incorpora a todos los niños con DI del segundo ciclo de ambas escuelas que quisieron participar en este estudio y cuya participación fue autorizada por su apoderado.

\subsection{Procedimiento de producción de información}

Se emplearon tres técnicas de producción de información: fotografía participativa, foto-elicitación y entrevista episódica. La primera técnica busca propiciar la investigación a través de la mirada de las personas, permitiéndoles que controlen los medios de representación fotográfica, situándolos como colaboradores en el proceso de construcción de conocimiento e incrementándoles su motivación (Rabadán y Contreras, 2014). Por tanto, se les instruyó a los participantes en el manejo de la cámara fotográfica y se les instó que autónomamente capturasen mediante diversas fotografías aquellas actividades escolares más valoradas durante su jornada escolar. Se recurrió a la fotografía participativa para capturar en instantáneas las actividades escolares socialmente valoradas respetando la perspectiva de los niños.

La foto-elicitación se basa en insertar una fotografía en una entrevista de investigación para estimular de mejor modo la memoria, permitiendo a los niños participar en la investigación aun cuando no dispongan de capacidades para discutir ideas abstractas (Cappello, 2005). Esta técnica se empleó antes de comenzar con las entrevistas, solicitándoles a los participantes que eligieran las fotografías que más les gustaban de entre todas las capturadas con objeto de exhibirlas mediante un proyector.

La tercera técnica de producción de información es la entrevista episódica. El elemento central de esta técnica es la invitación a presentar narraciones de las situaciones $y$, aunque el foco se pone en las narraciones, también es posible dar lugar a aclaraciones semánticas, diferenciándola de la entrevista narrativa (Flick, 2007). En esta entrevista, se buscaba que los niños narraran sus experiencias de participación en las actividades capturadas en fotografías y en otras que recordaran.

\subsection{Procedimiento de análisis de información}

Los textos resultantes de la transcripción de las entrevistas episódicas se analizaron mediante los lineamientos de la Teoría Fundamentada de Strauss y Corbin (2002). Esta disposición sigue la sugerencia de Flick (2007), quien estipula que los datos emanados de la entrevista episódica deben ser analizados bajo la codificación teórica. El propósito de la Teoría Fundamentada apunta a construir un esquema explicativo a partir de datos que integre varios conceptos relacionados. Este tipo de análisis permite pensar en abstracto distanciándose lo suficiente de los hechos particulares para que los resultados se puedan aplicar a otros miembros del grupo estudiado (Straus y Corbin, 2002). Puesto que el proceso analítico de información cualitativa es facilitado 
por el empleo del programa computacional Atlas.ti, especialmente al utilizar la Teoría Fundamentada, se optó por utilizar esta herramienta.

Los textos transcritos fueron revisados a través del microanálisis, el cual es una herramienta cualitativa enmarcada en la Teoría Fundamentada que consiste en un análisis minucioso de los datos por segmentos como una palabra, oración, línea y/o párrafo (Strauss y Corbin, 2002). El microanálisis está compuesto por la codificación abierta y axial, siendo la primera un proceso por medio del cual se construyen categorías y la segunda un proceso donde se relacionan las categorías elaboradas. De este modo, los fragmentos de entrevista que ilustran y condensan esas unidades de significado se vuelven relevantes para dar cuenta del objetivo analítico. Por ello, en el apartado de resultados se aportan fragmentos significativos de las entrevistas que sirven como ejemplos de las categorías presentadas, los que además son identificados con un nombre ficticio que representa a cada participante. Algunos de estos fragmentos contienen chilenismos, los cuales son acompañados con un sinónimo al interior de un paréntesis para facilitar su comprensión.

La codificación abierta se empleó al servicio del primer objetivo específico, tomando como unidades de análisis los episodios narrados para describir las características de las actividades que las contextualizaban. También se utilizó este tipo de codificación para el segundo objetivo específico a fin de generar categorías que representaran las ideas de los niños en torno a su participación en tales actividades. Estas categorías fueron relacionadas mediante la aplicación de la codificación axial, la que se distingue porque las relaciones se elaboran alrededor de alguna(s) categoría(s) (Strauss y Corbin, 2002). Las categorías que funcionaron de eje para esta codificación fueron los tipos de actividades socialmente valoradas desarrolladas.

\section{Resultados}

Los resultados se encuentran organizados en dos apartados, obedeciendo al orden de los dos objetivos específicos que dirigen esta investigación. En el primero, se presentan los tipos de actividades socialmente valoradas en el contexto escolar desde la perspectiva de los participantes de este estudio. En segundo término, se exponen las ideas de los niños con DI sobre su participación en el contexto de estas actividades socialmente valoradas.

\subsection{Tipos de actividades socialmente valoradas}

\subsubsection{Actividades de construcción de material físico y/o simbólico}

Los participantes del estudio describen como experiencias especialmente gratificantes los trabajos realizados por todos los estudiantes en la sala de clases, cuya finalidad correspondía a la construcción de objetos físicos y simbólicos. Estas actividades habitualmente eran llevadas a cabo bajo una modalidad grupal y, por lo tanto, se 
organizaba el curso en diversos grupos para que todos los estudiantes participaran. El profesor entregaba instrucciones generales y atendía dudas en el proceso.

La categoría “Actividades de construcción de material físico y/o simbólico” (en adelante actividades de construcción) incluye cinco subtipos de actividades, las que se diferencian por la naturaleza del producto físico y/o simbólico que se tenía por objetivo construir. Los subtipos de esta categoría son: producto físico, disertación, representación teatral y danza, elaboración de textos y construcción mixta. A continuación, se presenta un ejemplo que ilustra esta categoría:

\section{Fragmento 1 \\ ¿Publicidad? Sí, eso. Ab ya. Y teníamos que hacer eso y lo hicimos las cuatro. ¿Son el grupo? Sí, sí somos las únicas, nos juntamos. ¿Y tuvieron que hacer su propia publicidad? Sí. ¿Lo hicieron en un cartel? Sí, en un cartel [...] teníamos que actuar como ver eso así, de repente, y decir lo que era. [...] Me tocó decir que era un con- cierto. Ab era un concierto. Sí, que adónde era, cuánto costaba los lados donde uno se ganaba [ubicaba], la cancha, todo eso, los precios, eso me tocó, después teníamos que ir a comprar las entradas, eso me tocó con la Olga y con la Juana estaba ahí con la Renata atendiendo así (Emilia).}

\subsubsection{Juegos grupales}

Los niños con DI le atribuyen un significativo valor a los juegos grupales. Este tipo de actividad puede considerarse como socialmente valorado, puesto que según los estudiantes los juegos grupales son ampliamente practicados por un gran número de niños, niñas y adolescentes. Adicionalmente, es importante mencionar que, aunque los juegos descritos son muy diversos, la mayor parte poseen una formulación grupal, son voluntarios y están basados en la organización de los propios estudiantes, sin depender de la dirección del profesor u otro adulto. Por ejemplo:

\section{Fragmento 2}

¿Te acuerdas una situación en la escuela que te haya gustado mucho? Jugar con mis compañeros. [...] Cuando jugábamos a la pelota. ¿Recuerdas algún partido bien agradable para ti? Sí. Hace mucho tiempo ya. ¿Quién estaba jugando? Todos los hombres de mi curso [...] Dos equipos (Camilo).

\subsection{Ideas de los niños con discapacidad intelectual en el contexto de las actividades socialmente valoradas}

En este apartado se presentan las ideas que elaboran los niños a partir de su participación en actividades socialmente valoradas. Para tal efecto, estas ideas se despliegan en nueve categorías, algunas de cuales están asociadas a ambos tipos de actividad (construcción y juegos grupales) y otras a solo una de ellas. En la Figura 1 se presentan estas ideas y su relación con ambos tipos de actividad. 


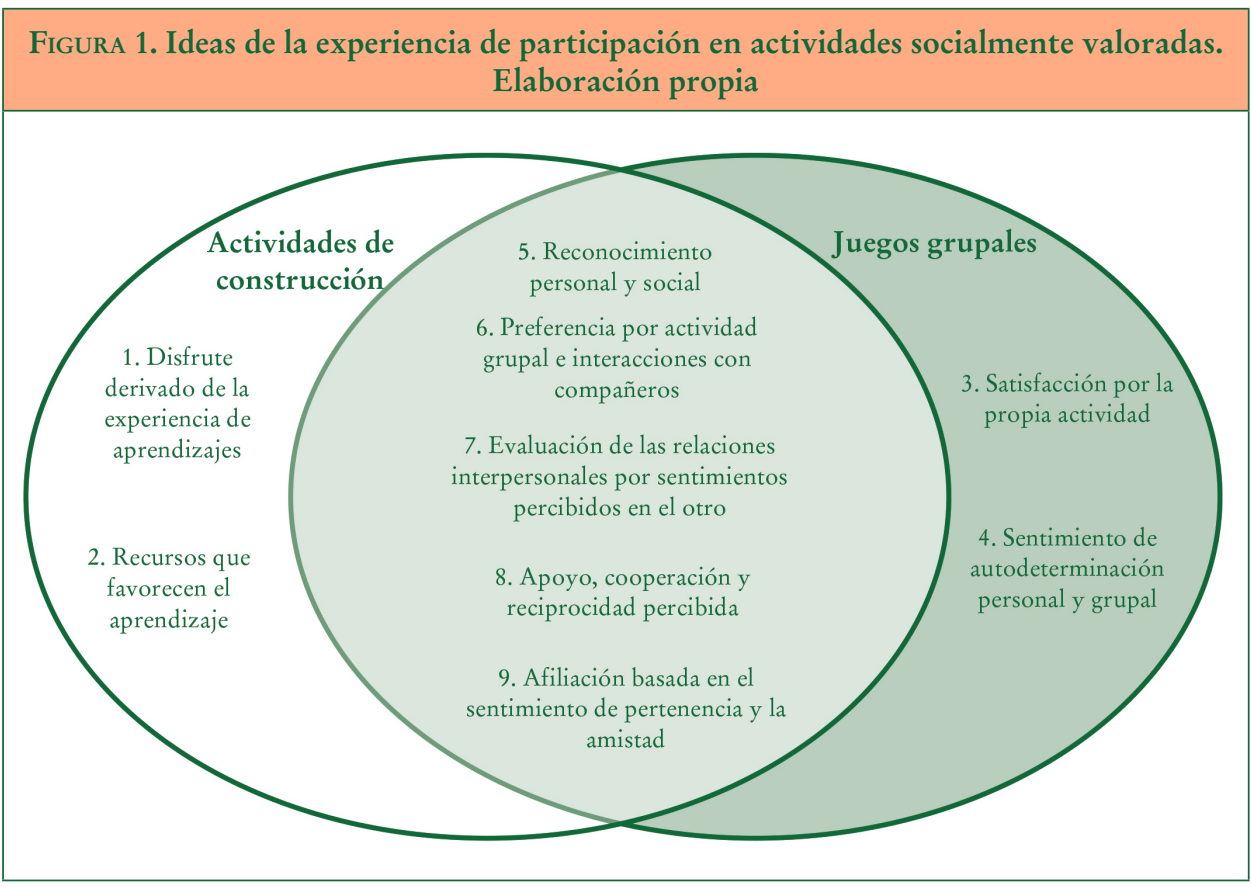

\subsubsection{Disfrute derivado de la experiencia de aprendizaje}

Los niños refieren que las actividades de construcción están caracterizadas por el aprendizaje esperado acerca de determinados contenidos y habilidades puntualizados por el profesor. Esta categoría refleja la satisfacción que indican los participantes al experimentar aprendizajes y percibir logros en términos escolares. El siguiente fragmento así lo muestra:

\section{Fragmento 3}

¿Te gustó esa actividad? Sí, estuvo bacán [excelente]. ¿Y qué cosa te gustó? No sé, toda la clase, es que matemática igual me gusta, cuando le agarró así o cuando sé algo, no sé, me divierto (Fabián).

\subsubsection{Recursos que favorecen el aprendizaje}

Los estudiantes afirman que reconocen múltiples recursos en su medio que les facilitan aprender en el contexto de las actividades de construcción. Estos recursos han sido clasificados en tres subcategorías: 1 ) uso de material concreto y equipo computacional, 2) capacidad de explicación y enseñanza del docente y 3) apoyo provisto por los compañeros. Estos atributos se encuentran presentes en los siguientes fragmentos: 


\section{Fragmento 4}

¿Te gustó? Sí, estuvo bacán [excelente]. ¿Quién estaba en tu grupo? Bien, bien no me acuerdo [...] ahora que me acuerdo no era parecido al papel lustre era unos triángulos con papel y teníamos que recortarlos y allí teníamos que ver cuánto media y ver las cosas así (Fabián).

\section{Fragmento 5}

¿Qué tenía ella que te gustaba? Porque nos explicaba bien. ¿Cómo es explicar bien? Si tú no entendías te volvía a explicar, te lo explicaba hasta que tú lo entendías y te lo explicaba de forma divertida, para reírse (Emilia).

\subsubsection{Satisfacción por la propia actividad}

Esta categoría hace referencia a la satisfacción experimentada por los estudiantes al participar en juegos grupales y a su motivación por tomar parte en estas actividades para divertirse. Por lo tanto, los juegos grupales tienen un fin en sí mismos al producir satisfacción solo por el acto de participar en ellos. Por ejemplo:

\section{Fragmento 6}

¿Por qué te gustan más? ¿Por qué te gusta física, por ejemplo? Porque a veces hacemos juegos, nos divertimos (Francisco).

\subsubsection{Sentimiento de autodeterminación personal y grupal}

Esta categoría hace referencia a que los niños con DI sienten que la participación en los juegos grupales se encuentra determinada por las decisiones que toman como individuos y como grupo de compañeros, sin depender de docentes o adultos. A continuación, un ejemplo:

\section{Fragmento 7}

Yo les copio jugando, pero por ejemplo ellos se tiran de la pasarela, yo no me voy a tirar o matar a una persona, eso no, ellos no me dicen pero les sigo jugando o si no yo juego y ellos empiezan todos, como que todo un grupo empezamos a jugar (Cecilia).

\subsubsection{Reconocimiento personal y social}

Esta categoría alude al reconocimiento que los participantes manifiestan vivenciar tanto en las actividades de construcción como en los juegos grupales. Este reconocimiento puede ser personal o social. El reconocimiento es personal al tomar consciencia de los propios logros y es social cuando emana del entorno social, como de compañeros y profesores. A continuación, se muestran tres ejemplos: 
JUGAR Y CONSTRUIR: EXPERIENCIAS DE PARTICIPACIÓN DE NIÑOS CON DISCAPACIDAD INTELECTUAL DE SEGUNDO CICLO BÁSICO EN DOS ESCUELAS MUNICIPALES EN CHILE

RODOLFO ISRAEL SOTO GONZÁLEZ Y MAURICIO ANDRÉS LÓPEZ CRUZ

\section{Fragmento 8}

¿Por qué te gustó? Porque podríamos hacer más cosas difíciles. Porque a mí me costó mucho la lámpara (Carla).

\section{Fragmento 9}

¿Te gusta el profe Oliver? Sí, él era el entrenador de nosotros mismos. ¿Conversó contigo? Sí, él me dijo que era bueno para jugar (Francisco).

\section{Fragmento 10}

Fui el más rápido [...] lo hice primero que todos. ¿Te sentiste valorado con eso? Sí, empezaron como a aplaudir (Pedro).

\subsubsection{Preferencia por actividad grupal e interacciones con compañeros}

Esta categoría refiere a la preferencia por la disposición grupal que comparten ambos tipos de actividad, la cual promueve un constante flujo de interacción entre compañeros. Los motivos que avalan esta preferencia destacan las múltiples formas de interacción que tienen lugar en esos escenarios de actividad. Esta categoría es ejemplificada con dos fragmentos:

\footnotetext{
Fragmento 11

¿Por qué te gustó más trabajar en equipo? Porque podí [puedes] decidir ¡ah! Ejemplo, te pueden, la profesora te puede pedir que hagas una maqueta sola y con equipo, y el equipo dice cómo se puede hacer, y uno da la respuesta y todo queda bonito y maravilloso porque trabajamos en equipo y es muy divertido (Susana).

\section{Fragmento 12}

¿Te gustaba? Sí. ¿Por qué? Era bien compartir con mis compañeros igual. Por ahí, jugaba (Camilo).
}

\subsubsection{Evaluación de las relaciones interpersonales por sentimientos percibidos en el otro}

La presente categoría refiere a la evaluación que realizan los participantes de las relaciones interpersonales que sostienen tanto con profesores como con sus compañeros. Estas relaciones se construyen principalmente a partir de las interacciones que permiten las actividades grupales. El aspecto característico de esta evaluación es que se fundamenta en los sentimientos percibidos que les dirigen otras personas. De esta manera, los profesores y compañeros que les demuestran simpatía, interés y alegría son preferidos y evaluados positivamente. En cambio, rechazan a aquellos profesores y compañeros que se enojan con ellos constantemente, manifestándolo a través de regaños y conductas de acoso. Ante estas circunstancias, los niños optan por alejarse y buscan trabajar de forma individual. En contraste, pueden tolerar de mejor manera regaños y burlas cuando les manifiestan simpatía de un modo regular. Los siguientes dos fragmentos ilustran esta categoría: 


\section{Fragmento 13}

La asignatura que más me gusta es ciencias naturales porque es muy simpática la profe. ¿Qué tiene de simpática? Que no es como la tía [profesora] Amelia, porque Ud. la abraza y es como un carácter que nadie le gusta. A la tía [profesora] Ramira le gusta que la abracen y es como más alegre. ¿Ella se enoja cuando hay que enojarse? Sí, cuando se portan mal, obvio que se enoja y todo. La tía [profesora] Amelia, si usted la abraza y le tiene cariño, a ella no le importa. Si ella fuera más simpática, más alegre, obvio que todos la querrían. Ni siquiera se ríe, la tía [profesora] Ramira se ríe. Es como más alegre, habla con nosotros (Fabiola).

\section{Fragmento 14}

Es que cuando iba en cuarto, yo quise quedar repitiendo porque ahí todos eran pesados y ahí nunca me podía concentrar en las pruebas, porque ahí hacían bullying (Francisco).

\subsubsection{Apoyo, cooperación y reciprocidad percibida}

Esta categoría concierne a la cooperación y el apoyo percibido por los niños como parte substancial de las interacciones sociales en las actividades grupales. De esta forma, la cooperación posee un carácter recíproco entre los participantes de dichas actividades y no solo tiene una naturaleza unidireccional a favor de los niños con DI. Por ejemplo:

\section{Fragmento 15}

Sí, el Max es como más, más estudioso, porque siempre cuando vamos a hacer las tareas yo lo ayudo un poco y él me ayuda a mí. ¿Y se ayudan? Sí. [...] Me, me ha dado de todo, cuando él tiene algo me da y yo igual le doy a él cuando tengo (Bruno).

\subsubsection{Afiliación basada en el sentimiento de pertenencia y la amistad}

Esta última categoría se trata de una de las consecuencias de las actividades socialmente valoradas. Esta consecuencia corresponde a la afiliación entre los estudiantes que toman parte de dichas actividades. En este sentido, se describe la afiliación desde el lazo de amistad que acerca afectivamente a dos estudiantes o a un grupo y también el sentimiento de pertenencia que se genera a partir de esa amistad. La base de la amistad y la pertenencia a un grupo es explicada por los estudiantes en función de las interacciones sociales que se han detallado como propias de las actividades socialmente valoradas. A continuación, esta categoría es ilustrada con dos ejemplos:

\section{Fragmento 16}

¿Qué tiene especial trabajar en equipo? Eh, divertirse y hacer nuevos amigos (Susana).

Fragmento 17

¿Cómo se generó esa amistad entonces? Cuando estábamos jugando a la pelota (Bruno). 
3.2.10. Esquema de las experiencias de participación

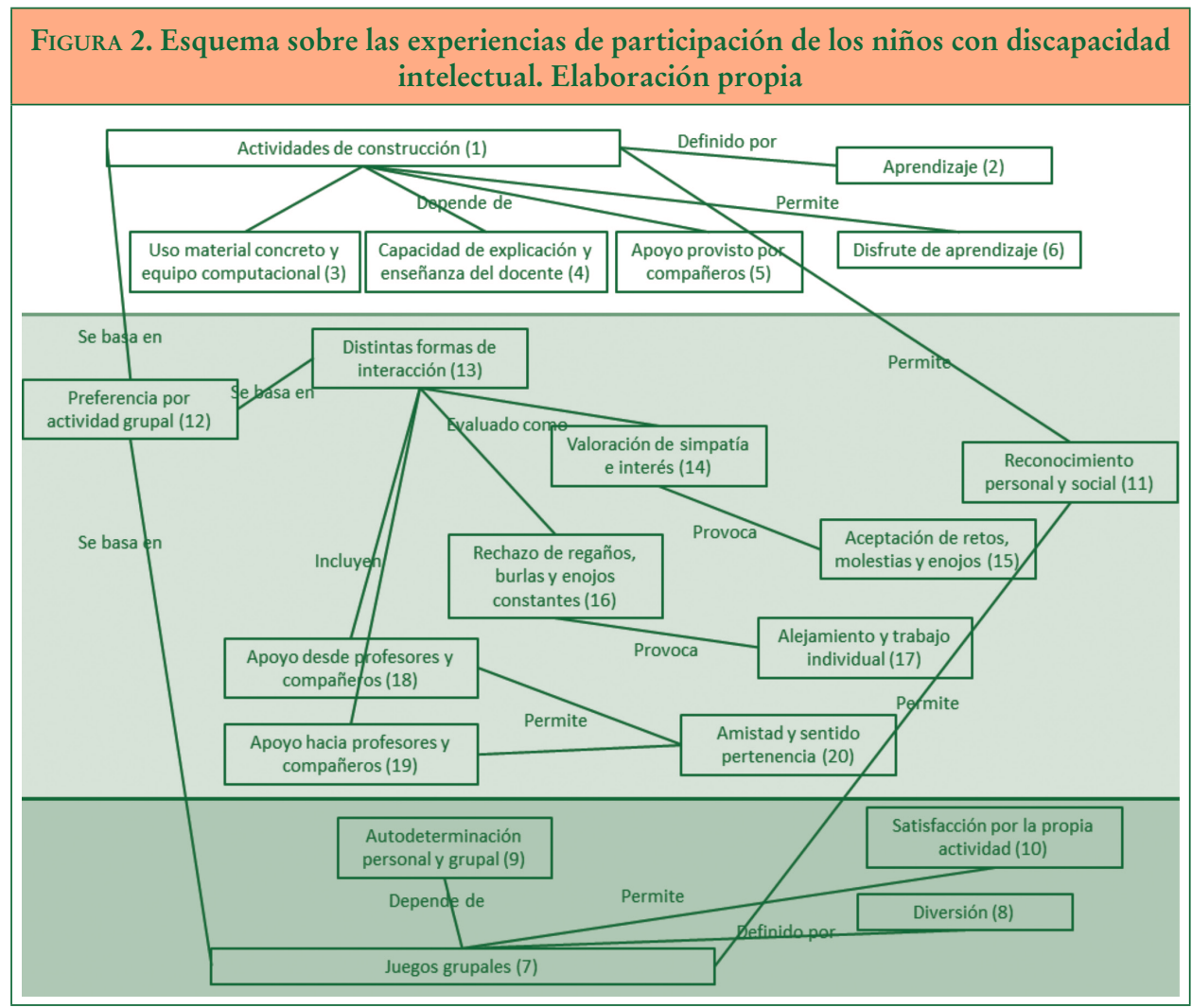

La Figura 2 es un esquema que incluye las ideas de las experiencias de participación de los niños con DI en las actividades socialmente valoradas y, de forma adicional, muestra las relaciones que guardan entre sí. Como se puede contemplar, ha sido asignado un número a cada etiqueta que representa una idea específica. Dichos números simbolizan el orden en que estas ideas y sus relaciones serán explicadas en los siguientes párrafos.

Las actividades de construcción (1) se hallan definidas principalmente por el aprendizaje que generan (2). Tal aprendizaje depende de la explicitación del docente que orienta la actividad y es un elemento que diferencia a los juegos grupales, ya que los entrevistados informan que estos últimos no derivan necesariamente a un aprendizaje.

Asimismo, el aprendizaje que define a la actividad de construcción es facilitado por distintos recursos: uso de material concreto y equipo computacional (3), capacidad de explicación y enseñanza del docente (4), junto con el apoyo provisto por los compañeros (5). Al experimentar el aprendizaje, los niños vivencian también un sentimiento de disfrute (6). 
Por otra parte, los juegos grupales (7) son definidos por los participantes de este estudio sobre la base de la diversión proporcionada por su participación. Esta diversión (8) que experimentan los niños al tomar parte de los juegos grupales les motiva a organizarse de un modo autoderminado con sus compañeros sin depender de la dirección del docente u otro adulto (9). Finalmente, los estudiantes participan en la actividad únicamente por la satisfacción que genera (10).

Tanto los juegos grupales como las actividades de construcción permiten que los participantes puedan obtener reconocimiento personal al darse cuenta de sus logros y obteniendo un sentimiento positivo y reconocimiento social, ya que son felicitados por sus compañeros y sus profesores (11).

Por otro lado, las actividades que han sido detalladas en este estudio comparten una modalidad grupal, la cual prefieren los niños (12). Este hecho aumenta la valoración social de estas actividades y es explicado por los participantes a partir de las distintas formas de interacción social que allí tienen lugar (13).

La intensa interacción social producida en el contexto de estas actividades permite que los participantes puedan evaluar las relaciones interpersonales que mantienen con sus compañeros y profesores. En particular, los niños informan que evalúan estas relaciones interpersonales fundamentalmente en base al tipo de afecto que los demás les dirigen. Por ejemplo, los participantes valoran la simpatía e interés de parte de algún compañero o profesor (14). Esta valoración permite que los niños puedan aceptar regaños y burlas de los demás (15).

Si el afecto que perciben de los demás es generalmente enojo demostrado en regaños y burlas, los niños con DI rechazarán estas relaciones interpersonales (16). Cuando se dan estos afectos negativos de manera constante, los niños revelan que optan por alejarse de estas personas y prefieren restringir estas relaciones interpersonales con trabajo individual (17).

Por su parte, el apoyo que proveen los profesores y compañeros (18) es parte integral de las interacciones interpersonales ocurridas en los escenarios de actividad, al igual que el apoyo que ofrecen los niños con DI hacia profesores y compañeros (19). En consecuencia, el apoyo es entendido por los estudiantes como recíproco entre los integrantes de las actividades y, por lo tanto, determinan relaciones de cooperación.

Las interacciones interpersonales en general y los apoyos recíprocos en particular que se dan en las actividades socialmente valoradas permiten que se construyan relaciones de amistad (20). Además, los niños anuncian que experimentan un sentimiento de pertenencia respecto a estos grupos de amigos. En este sentido, es menester hacer notar el relevante rol de la participación en estas actividades para propiciar sentimientos y relaciones de inclusión en los niños con DI.

\section{Discusión}

La presente investigación tuvo por objetivo comprender las experiencias de participación de niños con DI en el contexto escolar. Para alcanzar este objetivo se buscó identificar las actividades socialmente valoradas, contextualizando las experiencias de 
participación narradas y, posteriormente, se describieron las ideas de los estudiantes con DI sobre su participación en estas actividades. Los tipos de actividades socialmente valoradas que se identificaron fueron: actividades de construcción y juegos grupales.

La identificación de estas actividades permite mostrar alternativas que contrastan con formas tradicionales de intervención educativa, formas que implican una atención de los niños con DI en aulas separadas bajo el supuesto de que las dificultades de aprendizaje son esencialmente individuales y no pueden transformarse con la interacción escolar (López, Echeita y Martín, 2009). De esta manera, y al tratarse de recursos ordinarios que influyen a todo el alumnado, estas actividades pueden emplearse para limitar el uso de medidas especiales de atención reservadas para unos pocos (Durán y Vidal, 2004). La promoción de estas actividades implicaría también el disfrute del derecho de los niños con DI a una educación inclusiva, la cual debe darse en el contexto de su propia comunidad y con sus iguales (ONU, 2006a).

Las actividades escolares estudiadas son altamente significativas y motivantes para los niños con DI por su formato grupal y cooperativo, las interacciones sociales que proveen y el reconocimiento personal y social vivenciado en las mismas. Esta motivación tiene importancia educativa, ya que el deseo facilita que los niños puedan significar a las personas, los instrumentos materiales y sistemas simbólicos como mediadores o recursos para el aprendizaje (Pea, 2001). Esta significación es también fomentada mediante la inscripción de los apoyos en los escenarios de actividad, permitiendo que el contexto sociohistórico otorgue significado a los recursos y acciones (Wenger, 2002). En esta línea, la principal fuente de apoyo identificada en las actividades socialmente valoradas son las relaciones de cooperación entre pares, recurso tradicionalmente desaprovechado para propiciar el aprendizaje (Ainscow, 2001; Durán y Vidal, 2004). No obstante, la cooperación es sugerida como estrategia fundamental para dar respuesta a la diversidad en el contexto regular (Pujolàs, 2010).

Al parecer, los objetos elaborados en las actividades de construcción también funcionan como apoyos. De esta forma y al igual que este estudio, Campos (2015) identificó una relación entre la cooperación de los estudiantes y las actividades cuyo objetivo radicaba en la construcción de objetos físicos y/o simbólicos. Además de alentar la cooperación, las construcciones juegan un significativo papel en la consciencia de los niños con DI respecto a sus aprendizajes, ya que los niños perciben el alcance de aprendizajes mediante la construcción de objetos. Es posible suponer que como los niños con DI poseen limitaciones generalmente en el pensamiento abstracto (AADID, 2011), los mediadores de carácter más concreto permiten auxiliar de mejor modo su cognición, como lo plantea Pea (2001) al examinar el rol de artefactos y herramientas. En esta dirección, Cevik y Uredi (2016) encontraron que estudiantes con DI mejoraron su rendimiento académico y sus actitudes hacia la ciencia mediante un proyecto basado en construcción de maquetas.

Dado que los estudiantes manifiestan que participan en los juegos grupales por el goce producido bajo una organización autodeterminada, es posible indicar que funcionan como apoyos especialmente en el plano motivacional. Estos resultados, siguiendo a Ryan y Deci (2000), permiten sostener que los niños participan en los 
juegos grupales por motivación intrínseca y por motivación extrínseca con un locus interno de causalidad percibido. Por motivación intrínseca, ya que la actividad por sí misma les produce una satisfacción inherente. También por motivación extrínseca, puesto que la mayor parte de los juegos descritos disponían de reglas y los niños se sujetaban a estas de forma autónoma. Esta observancia voluntaria de reglas favorece la capacidad de autorregulación de los niños con DI, la cual es una función psicológica superior y como tal permite dirigir otros procesos psicológicos (Vygotsky, 1978).

Por otro lado, y de acuerdo a las experiencias de los niños con DI, los escenarios de actividad descritos cumplen un importante rol para mejorar su calidad de vida. En efecto, esta investigación muestra que los estudiantes con DI desarrollan relaciones en grupos de amistad y un sentimiento de pertenencia hacia estos, elementos fundamentales en el mejoramiento de la calidad de vida (Schalock y Verdugo, 2007). Además, los resultados muestran un significativo nivel de bienestar emocional, ya que los relatos de las experiencias de participación se caracterizan por papeles asumidos que derivaban en logros y reconocimientos. Por ende, estos relatos permiten a los estudiantes percibirse no solamente en función de características patológicas, sino por sus potencialidades, virtudes y logros. Estas percepciones positivas son consistentes con la búsqueda de la inclusión educativa basada en la celebración de las diferencias (Pujolàs, 2010).

Los antecedentes descritos permiten identificar variados apoyos presentes en estas actividades socialmente valoradas. Estos apoyos no están necesariamente diseñados para los niños con DI y, por lo tanto, favorecen su acceso al currículo general. La importancia del acceso universal radica en que su alcance disminuye la necesidad de apoyos intrusivos que podrían favorecer una imagen negativa al estudiante y reforzar con ello su estigmatización (Wehmeyer, 2009). De esta manera, estos apoyos son coherentes con la lógica del diseño universal para el aprendizaje, el cual defiende la idea de gestar un currículo para todos mediante la flexibilización de los medios para dar lugar al aprendizaje (CAST, 2008). Por lo tanto, y tomando como base la experiencia de los estudiantes con DI, estas actividades, y los apoyos presentes en ellas, contribuyen al objetivo de la pedagogía inclusiva por hallar "oportunidades de aprendizaje ricas y que sean suficientemente accesibles para todos, de tal forma que todos los alumnos sean capaces de participar en la vida del aula” (Florian, 2013: 34).

El material discutido previamente puede sintetizarse en tres conclusiones. En primer término, es posible caracterizar la participación en el contexto escolar de los niños con DI destacando el importante rol que juegan el reconocimiento, la amistad, la satisfacción, el sentimiento de pertenencia y la cooperación en sus experiencias. En segundo término, las experiencias de participación adquieren una significación relevante desde la perspectiva de los estudiantes con DI en relación con el aprendizaje, la calidad de vida, el desarrollo y la motivación. Por último, las experiencias de participación de los niños con DI se dan, y son potenciadas, con recursos de carácter universal, es decir, pueden también ser aprovechados por todos los estudiantes.

Estas conclusiones deben considerarse bajo el contexto de ciertas limitaciones que presenta este estudio. En primer lugar, los participantes de este estudio pertenecían a segundo ciclo de educación básica del sistema escolar chileno, dejando fuera otros 
JUGAR Y CONSTRUIR: EXPERIENCIAS DE PARTICIPACIÓN DE NIÑOS CON DISCAPACIDAD INTELECTUAL DE SEGUNDO CICLO BÁSICO EN DOS ESCUELAS MUNICIPALES EN CHILE RODOLFO ISRAEL SOTO GONZÁLEZ Y MAURICIO ANDRÉS LÓPEZ CRUZ

niveles educativos. En segundo lugar, los estudiantes únicamente presentaban el diagnóstico de discapacidad intelectual y, por ende, no se incorporaron niños que además tuvieran otra discapacidad. En tercer lugar, las experiencias de participación han sido reconstruidas desde la memoria de los niños del pasado cercano y lejano, sin indagar en la evolución de las experiencias en el presente.

Partiendo de estas limitaciones y los resultados de la presente investigación, futuros estudios podrían enfocarse en recoger las experiencias de participación de niños con DI de otros niveles educativos y con otras discapacidades. Asimismo, sería pertinente enriquecer la información producida mediante la aplicación de técnicas de estudio asociadas a la etnografía y la investigación-acción para analizar el proceso y evolución de la experiencia en torno a diversas actividades. Otra proyección corresponde a comprender, también, las experiencias de segregación y exclusión de los niños con DI con la finalidad de comparar su perspectiva con la analizada en esta investigación. Por último, sería interesante indagar longitudinalmente en el desarrollo y aprendizaje futuro de los niños con DI que disfrutaron de abundantes experiencias de participación en su proceso escolar.

\section{Referencias bibliográficas}

Ainscow, M. (2001). Desarrollo de escuelas inclusivas. Ideas, propuestas y experiencias para mejorar las instituciones escolares. Madrid: Narcea.

Asociación Americana de Discapacidades Intelectuales y del Desarrollo (2011). Discapacidad Intelectual: Definición, Clasificación y Sistemas de Apoyo. Madrid: Alianza.

Black-Hawkins, K., Florian, L. y Rouse, M. (2008). Achievement and Inclusion in Schools and Classrooms: Participation and Pedagogy. Artículo presentado en la conferencia de British Educational Research Association. Universidad Heriot Watt. Edinburgh.

Bоoтн, T. y Ainscow, M. (2011). Guía para la inclusión educativa: Desarrollando el aprendizaje y la participación en las escuelas. Reino Unido: CSIE

Campos, M. P. (2015). Aprendizaje a través de la cooperación entre pares desde la perspectiva de los estudiantes para el desarrollo de escuelas inclusivas (Tesis de Magíster). Universidad de Chile, Santiago de Chile.

Cappello, M. (2005). Photo interviews: Eliciting data through conversations with children. Field Methods, 17 (2), 170-182.

Centro para la Tecnología Especial Aplicada (2008). Universal design for learning guidelines version 1.0. Wakefield, MA. Recuperado de: http://web.uam.es/personal_pdi/stmaria/sarrio/ DOCENCIA/ASIGNATURA\%20BASES/LECTURAS\%20ACCESIBLES $\% 20 \mathrm{Y} \% 20$ GUIONES\%20DE\%20TRABAJO/Diseno\%20Universal\%20de\%20Aprendizaje.pdf.

CEviK, M. y UREDI, F. (2016). Effects of the project-based learning on academic achievement and attitude of students with mild intellectual disability in life science course. International Journal of Learning and Teaching, 8 (2), 90-99.

Comité de los Derechos del NiÑo (2009). Observación General N. ${ }^{\circ} 12$ sobre el derecho del niño a ser escuchado. Recuperado de: www2.ohchr.org/english/bodies/crc/docs/.../CRCC-GC-12_sp.doc.

Durán, D. y Vidal, V. (2004). Tutoría entre iguales. De la teoría a la práctica. Barcelona: Graó.

(C) Ediciones Universidad de Salamanca / CC BY-NC-ND

Siglo Cero, vol. 48 (2), n. ${ }^{\circ}$ 262, 2017, abril-junio, pp. 61-79 
JUGAR Y CONSTRUIR: EXPERIENCIAS DE PARTICIPACIÓN DE NIÑOS CON DISCAPACIDAD INTELECTUAL DE SEGUNDO CICLO BÁSICO EN DOS ESCUELAS MUNICIPALES EN CHILE RODOLFO ISRAEL SOTO GONZÁLEZ Y MAURICIO ANDRÉS LÓPEZ CRUZ

European Agency for Development in Special Needs Education (2011). Participation in Inclusive Education: A Framework for Developing Indicators. Dinamarca: European Agency for Development in Special Needs Education.

Fielding, M. (2011). La voz del alumnado y la inclusión educativa: una aproximación democrática radical para el aprendizaje intergeneracional. Revista Interuniversitaria de Formación de Profesorado, 25 (1), 31-61.

FLICK, U. (2007). Introducción a la investigación cualitativa. Madrid: Morata.

FLORIAN, L. (2013). La educación especial en la era de la inclusión: ¿El fin de la educación especial o un nuevo comienzo? Revista Latinoamericana de Inclusión Educativa, 7 (2), 27-36.

Fondo Nacional de Discapacidad (2005). Primer estudio nacional de la discapacidad en Chile. Recuperado de: http://www.ine.cl/canales/chile_estadistico/encuestas_discapacidad/ $\mathrm{pdf} / \mathrm{reultados} 3 . \mathrm{pdf}$.

FundACIÓN CHILE (2013). Análisis de la implementación de los programas de integración escolar (PIE) en establecimientos que han incorporado estudiantes con necesidades educativas especiales transitorias (NEET). Recuperado de Ministerio de Educación: http://www.mineduc.cl/ usuarios/edu.especial/doc/201402101719500.InformeEstudioImplementacionPIE2013.pdf.

JUNAEB (2005). Sinae sistema nacional de asignación con equidad para becas Junaeb: Una nueva visión en la construcción de igualdad de oportunidades en la infancia. Santiago de Chile: Gobierno de Chile.

JUNAEB (2015). Prioridades 2015 con IVE SINAE básica media y comunal. Chile. Base de datos recuperada de: http://www.junaeb.cl/ive.

LansDown, G. (2005). La evolución de las facultades del niño. Italia: Unicef.

López, M., Echeita, G. y Martín, E. (2009). Concepciones sobre el proceso de inclusión educativa de los alumnos con discapacidad intelectual en la educación secundaria obligatoria. Cultura y Educación, 21 (4), 485-496.

López, M., Valdivia, A. y Fernández, R. (2016). Producciones narrativo-visuales y voz de los y las estudiantes: indagación sobre los significados de participación en niños, niñas y jóvenes en escuelas municipales en Chile. FQS, 17 (1).

López, V. (2015). Discriminación y segregación: Efectos de la integración escolar sobre los proyectos de vida de estudiantes egresados de escuelas municipales que participaron en proyectos de integración escolar. Revista Latinoamericana de Educación Inclusiva, 8 (2), 69-83.

López, V., Julio, C., Pérez, M. V., Morales, M. y Rojas, C. (2014). Barreras culturales para la inclusión: políticas y prácticas de integración en Chile. Revista de Educación, 363 (1), 256-281.

Ministerio de Educación (2009a). Decreto Nro. 170. Fija normas para determinar los alumnos con necesidades educativas especiales que serán beneficiarios de las subvenciones para educación especial. Recuperado de Ministerio de Educación: http://www.mineduc.cl/usuarios/edu.especial/doc/201304231500550.DEC200900170.pdf.

MiNISTERIO DE EDUCACIÓN (2009b). Manual de orientaciones y apoyo a la gestión (Directores y Sostenedores). Recuperado de Ministerio de Educación: http://www.mineduc.cl/usuarios/ edu.especial/doc/201405071255480.ManualOrientacionesPIE.pdf.

Organización de las Naciones Unidas (1986). Convención sobre los Derechos del Niño. Recuperado de: https://www.unicef.es/sites/www.unicef.es/files/CDN_06.pdf.

Organización de las Naciones Unidas (2006a). Los principales tratados internacionales de derechos bumanos. Recuperado de: http://www.ohchr.org/Documents/Publications/CoreTreatiessp.pdf.

(C) Ediciones Universidad de Salamanca / CC BY-NC-ND

Siglo Cero, vol. 48 (2), n. ${ }^{\circ}$ 262, 2017, abril-junio, pp. 61-79 
JUGAR Y CONSTRUIR: EXPERIENCIAS DE PARTICIPACIÓN DE NIÑOS CON DISCAPACIDAD INTELECTUAL DE SEGUNDO CICLO BÁSICO EN DOS ESCUELAS MUNICIPALES EN CHILE RODOLFO ISRAEL SOTO GONZÁLEZ Y MAURICIO ANDRÉS LÓPEZ CRUZ

Organización de las Naciones Unidas (2006b). Convención sobre los Derechos de las Personas con Discapacidad. Recuperado de: http://www.un.org/esa/socdev/enable/documents/ tccoonvs.pdf.

Pea, R. (2001). Prácticas de inteligencia distribuida y diseños para la educación. En G. SALOMÓN (Comp.), Cogniciones distribuidas. Buenos Aires: Norma.

Pujolàs, P. (2010). Aprender juntos alumnos diferentes, los equipos de aprendizaje cooperativo en el aula. Barcelona: Octaedro.

RabadÁn, A. y Contreras, P. (2014). La Fotografía Participativa en el contexto socio-educativo con adolescentes. Revista Interdisciplinar de Ciencias de la Comunicación y Humanidades, 10, 143-156.

Rogoff, B. (1997). Los tres planos de la actividad sociocultural. En J. WerTsCH, P. DEL Río y A. Álvarez (Comps.), La mente sociocultural: Aproximaciones teóricas y aplicadas (pp. 111-128). Madrid: Astáviz.

Ryan, R. y Deci, E. (2000). Self-determination theory and the facilitation of intrinsic motivation, social development and well-being. American Psychologist, 55, 68-78.

Schalock, R. y Verdugo, M. Á. (2007). El concepto de calidad de vida en los servicios y apoyos para personas con discapacidad intelectual. Revista Española sobre Discapacidad Intelectual, 38 (4), 21-36.

Silverman, D. (2000). Doing Qualitative Research. A Practical Handbook. Londres: Sage.

Strauss, A. y Corbin, J. (2002). Bases de la Investigación Cualitativa. Medellín: Editorial de la Universidad de Antioquia.

UNICEF (2013). Estado mundial de la infancia: niños y niñas con discapacidad. Recuperado de: http://www.Lansdown, G. (2005). La evolución de las facultades del niño. Italia: Unicef.w.unicef.org/ecuador/SPANISH_SOWC2013_Lo_res.pdf

Wehmeyer, M. (2009). Autodeterminación y la tercera generación de prácticas de inclusión. Revista de Educación, 349, 45-67.

Wenger, E. (2002). Comunidades de Práctica: aprendizaje, significado e identidad. España: Paidós.

Verdugo, M. Á., Navas, P., Gómez, L. E. y Schalock, R. L. (2012). The concept of quality of life and its role in enhancing human rights in the field of intellectual disability. Journal of Intellectual Disability Research, 56 (11), 1036-1045.

Verdugo, M. Á., Schalock, R., Thompson, J. y Guillén, V. (2013). Discapacidad intelectual: definición, clasificación y sistemas de apoyo. En M. Á. Verdugo y R. Schalock (Comps.), Discapacidad e Inclusión: manual para la docencia (pp. 89-110). Salamanca: Amarú.

Villalobos, B., Carrasco, C., López, V., Bilbao, M. A., Morales, M., Ortiz, S., Olavarría, D., Ascorra, P. y Del Castillo, A. A. (2014). Inclusión y violencia: Prevalencia de victimización entre pares en estudiantes que participan en Programas de Integración Escolar. Revista Latinoamericana de Educación Inclusiva, 8 (2), 161-178.

Vygotsky, L. S. (1978). Pensamiento y lenguaje. Madrid: Paidós.

Woolfson, R., Harker, M., Lowe, D., Sheilds, M. y Mackintosh, H. (2007). Consulting with children and young people who have disabilities: views of accessibility to education. British Journal of Special Education, 34 (1), 40-49. 